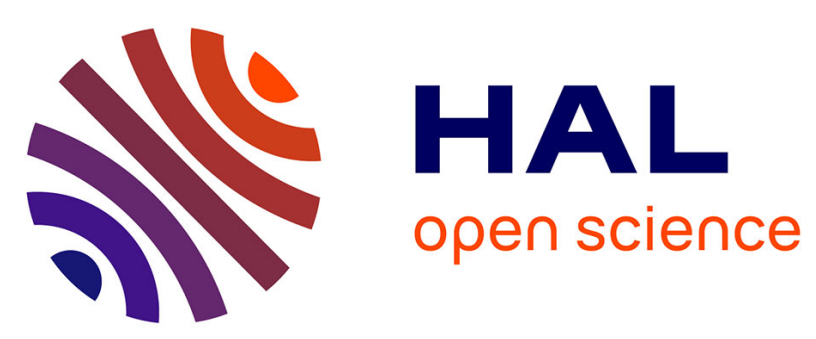

\title{
A Domain Specific Language for Orchestrating User Tasks Whilst Navigation Web Sites
}

\author{
Sergio Firmenich, Gustavo Rossi, Marco Winckler
}

\section{To cite this version:}

Sergio Firmenich, Gustavo Rossi, Marco Winckler. A Domain Specific Language for Orchestrating User Tasks Whilst Navigation Web Sites. 13th International Conference on Web Engineering (ICWE 2013), Jul 2013, Aalborg, Denmark. pp. 224-232. hal-01239731

\section{HAL Id: hal-01239731 \\ https://hal.science/hal-01239731}

Submitted on 8 Dec 2015

HAL is a multi-disciplinary open access archive for the deposit and dissemination of scientific research documents, whether they are published or not. The documents may come from teaching and research institutions in France or abroad, or from public or private research centers.
L'archive ouverte pluridisciplinaire HAL, est destinée au dépôt et à la diffusion de documents scientifiques de niveau recherche, publiés ou non, émanant des établissements d'enseignement et de recherche français ou étrangers, des laboratoires publics ou privés. 


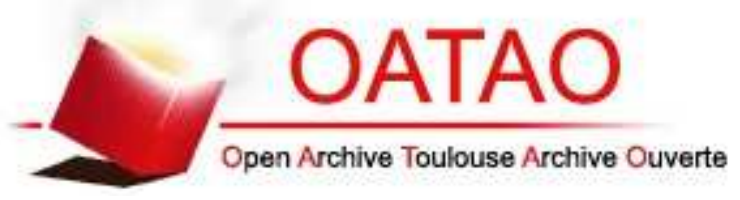

\section{Open Archive TOULOUSE Archive Ouverte (OATAO)}

OATAO is an open access repository that collects the work of Toulouse researchers and makes it freely available over the web where possible.

This is an author-deposited version published in : http://oatao.univ-toulouse.fr/ Eprints ID : 12486

The contribution was presented at ICWE 2013 :

http://icwe2013.webengineering.org/

Official URL: http://dx.doi.org/10.1007/978-3-642-39200-9_20

To cite this version : Firmenich, Sergio and Rossi, Gustavo and Winckler, Marco Antonio A Domain Specific Language for Orchestrating User Tasks Whilst Navigation Web Sites. (2013) In: 13th International Conference on Web Engineering (ICWE 2013), 8 July 2013 - 12 July 2013 (Aalborg, Denmark).

Any correspondance concerning this service should be sent to the repository administrator: staff-oatao@listes-diff.inp-toulouse.fr 


\title{
A Domain Specific Language for Orchestrating User Tasks Whilst Navigation Web Sites
}

\author{
Sérgio Firmenich ${ }^{1,2}$, Gustavo Rossi ${ }^{1,2}$, and Marco Winckler ${ }^{3}$ \\ ${ }^{1}$ LIFIA, Facultad de Informática \\ ${ }^{2}$ Universidad Nacional de La Plata and Conicet Argentina \\ ${ }^{3}$ IRIT, Université Paul Sabatier, France \\ \{gustavo, sergio.firmenich\}@lifia.info.unlp.edu.ar, \\ winckler@irit.fr
}

\begin{abstract}
In this paper we claim that there are a lot of processes over Web applications that require a high level of coordination between individuals and tasks featuring procedures. We propose hereafter a Domain Specific Language (DSL) for describing the asynchronous orchestration users' tasks including manual users' tasks (i.e. simple instructions that tell users what to do during the navigation) and automated tasks (i.e. tasks that can be partially or completely automated by client-side scripts). The approach is illustrated by examples and a case study showing the tools, for which an empiric evaluation is presented.
\end{abstract}

Keywords: task and process modeling, Web application, Web augmentation.

\section{Introduction}

Although Web navigation was regarded in the past as a solitary activity, nowadays, many users are engaged in repetitive and collaborative activities that are supported by uncountable Web applications [6]; for example booking a seat in a flight or explaining friends how to book a seat next yours in a flight... Moreover, many of these tasks involve dealing with different Web sites, which run independently with no support to the actual users' concern [4].

This lack of integration of different Web resources has motivated the development of mash-ups tools that are able merge into a specialized applications a set resources that are scattered among different Web sites [8]. The problem is that mash-up are used straightforward, when most of tasks users perform are volatile and do not really require the creation of a new an entirely new applications.

The integration of data across applications can also be done by Web augmentation artifacts, which perform interventions over Web applications DOMs. Some Web augmentation approaches [1][4] aim to support users task by adapting the Web pages visited accordingly.

In this paper we propose a Domain Specific Language (DSL) for describing procedures that are aimed to orchestrate user tasks over multiple Web sites. It supports flexible process modeling by allowing users to combine manual task and automated tasks from a repertoire of patterns of tasks performed over the Web. Whilst manual tasks can be regarded as simple instructions, automated tasks correspond to Web 
augmentation [4] tools (i.e. augmenters). The approach is duly illustrated by a case study describing a trip planning over the Web.

The rest of the paper is organized as follows: section 2 motivates and presents related works; section 3 introduces the approach. Section 4 present the DSL followed by the corresponding tool support (section 5). In section 5 we also present a comparative study using our tools; and lately section 6 presents the conclusions and future work.

\section{Motivation and Related Work}

Web Augmentation is not a new concept, and it is becoming really important from the point of view of users, who are expecting new kinds of mechanisms for personalizing their experience while navigating the Web. Large communities of scripting such as GreaseMokey prove the value of this technique. There are other similar approaches. Mashup tools, for instance, have the same final goal: improve the users' experience. Neither mash-ups nor existing Web augmentation techniques provide a definite and flexible solution for supporting users tasks. Here, we compare our approach with others DSL/tools for supporting users tasks.

Some approaches allow users to specify the steps involved in certain tasks in order to repeat these steps later. For example CoScripter [4] records the user interactions (based on DOM events) and then the user may reproduce the same steps automatically. Other approaches define DSLs that aim to help to automate tasks. For instance, ChickenFoot [1] extends JavaScript with new sentences (e.g. "click()", "enter()", etc.). In this way, to develop a script for automating Web use is easier. Both ChickenFoot and CoScripter are powerful approaches but these do not contemplate changes in the process, since it is completely DOM-dependent. With the same philosophy we can mention Selenium [7], which can be used for this task automation, although it was originally defined for testing. While all these approaches may help users by allowing them to automate only primitive tasks, our approach mixes these with augmentation ones, which adapt Web pages accordingly to the current user tasks. It implies that not only repetitive processes may be defined but complex scenarios of adaptation. Besides that, the manual execution of certain tasks gives the control to users. In this way, sensitive tasks (for example payments, or sensible information use) are not performed by automatic tasks in which users may not trust.

\section{Overview of Our Approach for Orchestration of Web Tasks}

This section provides a view at glance of our approach and the type of users' tasks supported which include: primitive and augmentation tasks.

We refer as primitive tasks to a basic set of tasks that are already supported by the Web browser. These tasks include actions such as "go to a Web page", "fill in a form", etc. Primitive tasks used in our approach are heavily inspired by previous works that have already proposed a taxonomy for these user tasks [7].

For us, augmentation tasks are those ones that require advanced scripts programming (based on Web augmentation techniques) to be executed over the Web browser. Some 
of these tools are able to perform changes in DOM's changing Web pages on the client side. In previous work [4] we have developed a set of Web augmentation tools, called augmenters, using the CSN framework. The CSN framework is a tool that supports the development of scripts aimed to adapt Web sites accordingly to the actual users' concern. Augmenter are integrated into the Web browser via the framework. Once installed, augmenters are accessible to the user via a contextual menu. The framework has two main user roles: i) developers: are users with programming skills who can extend the framework by creating augmenters; ii) final users: who use augmenters to improve their performance whilst navigating the Web. For example, Figure 1 shows the activation of the augmenter DataCollection used to collect data from Web pages. The data collected is presented as a kind of floating post-it called Pocket. In the example the user is collecting point of interest under the name of "PoI". As we shall, the collection of Web page data requires an advanced script (i.e. an augmenter), it modifies the DOM page (by creating a floating DIV element) and extend what users can do over a Web page (i.e. create electronic post-its); so that when a user runs the DataCollection augmenter he in fact performing an augmentation task.

\begin{tabular}{|c|c|c|c|}
\hline $\begin{array}{l}\text { lovias integradas y protegidas, } \\
\text { tac } \quad x, \text { mnibus } d\end{array}$ & $\begin{array}{l}\text { Undo adaptation augmenter } \\
\text { Add as model concept... }\end{array}$ & transporte. ${ }^{157158}$ & autopi \\
\hline Pol & DataCollectors & Put it into Pocket & \\
\hline $\begin{array}{l}\text { Buenos Aires } \\
\text { ta, } \\
\text { utopista Buenos Aires - La Plat }\end{array}$ & $\begin{array}{l}\text { Inspect Element with Firebug } \\
\text { la Autopista Ricchieri, el Acceso Oeste y el A }\end{array}$ & $\begin{array}{l}\text { Create instance into Pocket... } \\
\text { Add DOM instance into Pocket... }\end{array}$ & \\
\hline
\end{tabular}

Fig. 1. Example of the use of the augmenter DataCollection

Augmenters can also be used in combination to create complex sequences of tasks. Figure 2 shows the combined execution of augmenters. In this example a user executes the augmenter CreateGoogleMapsLink from the Pocket element (2.a). This action adds an anchor to GoogleMaps next to each occurrence of the concept "PoI" (2.b) that can then navigated to the corresponding GoogleMaps web site (2.c).

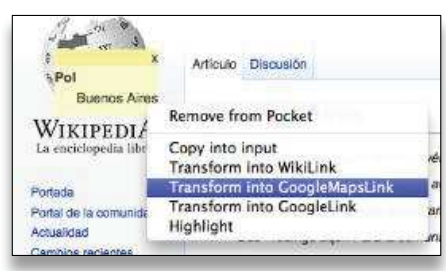

Fig. 2.a. Triggering augmenter using GoogleMapLink

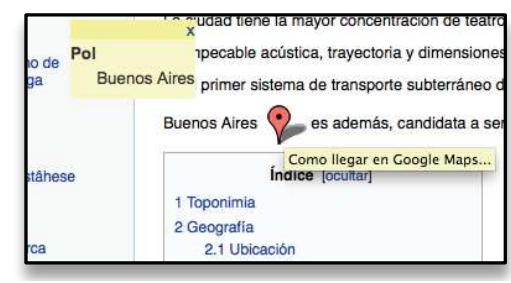

Fig. 2.b. Adaptation performed by the augmenter GoogleMapLink

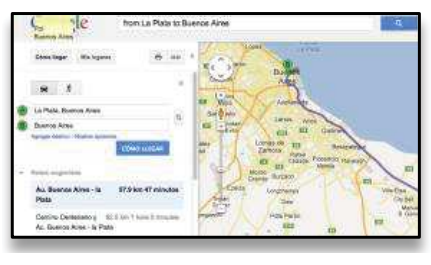

Fig. 2.c. Navigation to GoogleMaps

\subsection{Overview of the Approach}

The goal is to allow users to create complex processes, called procedures, by composing primitive and augmentation tasks. The composition is a sequence of tasks formalized by a DSL and stored as a XML file. A dedicated tool parses that XML file and 
executes the procedures on the Web browser. Figure 3 provides a view at glance of the approach. As we shall see, the approach include three phases, as follows:

- Definition of tasks: it concerns the inclusion of task to be composed. This phase requires skilled Web developers who program augmenters. This is technically demanding, but the work should be done once and it will benefit all users. Nonetheless, the framework provides a large set of both primitive and augmentation tasks.

- During Composition phase, users create a sequence of tasks available in the repository, which is exported by the factory and defined by the means of a DSL describing all tasks in the procedure. This artifact, defined by the DSL, may be shared with other users in order to support them in the accomplishment of the same task.

- Execution: this phase features a player that is concerned by the execution of the procedure previously encoded by the DSL.

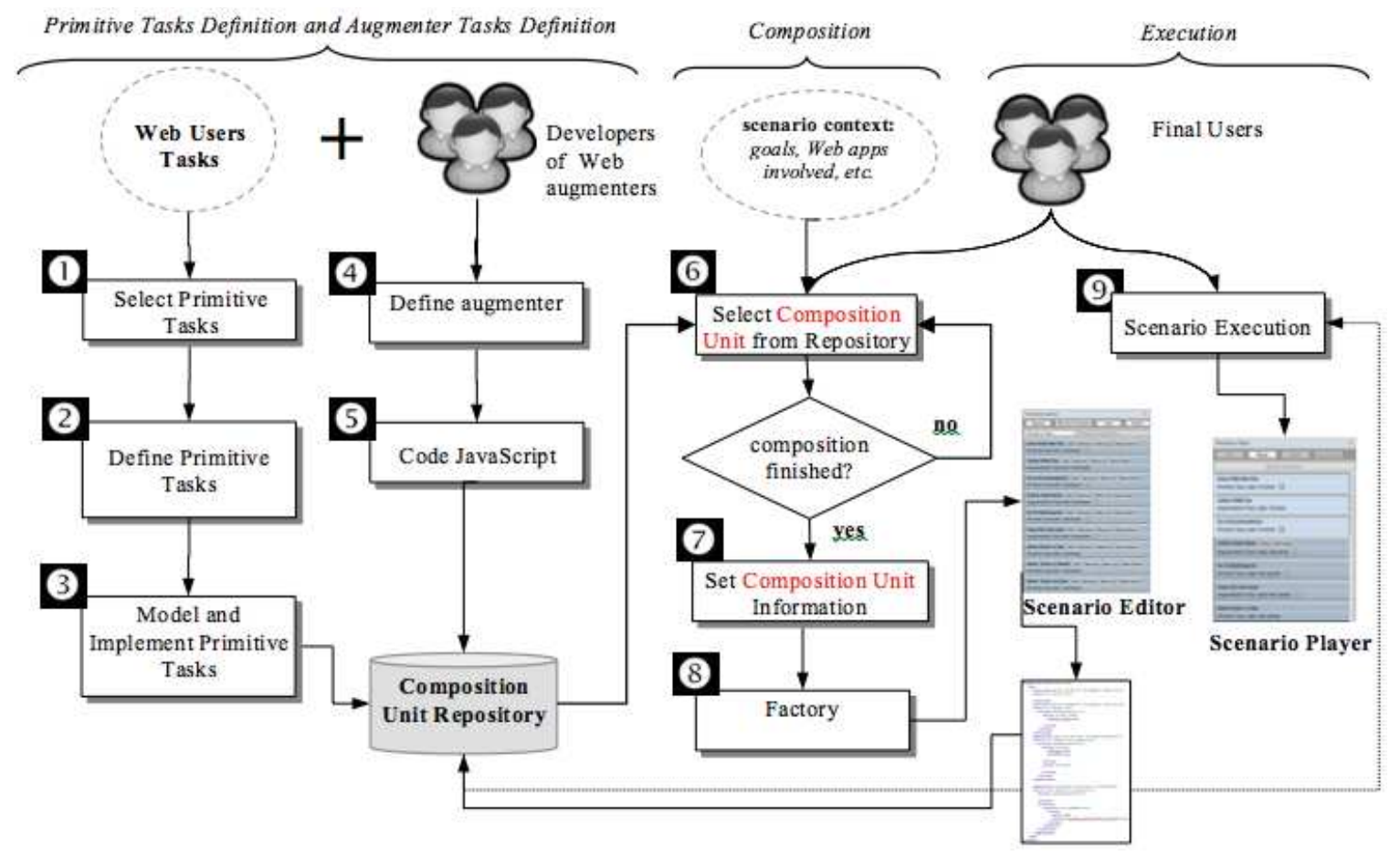

Fig. 3. Overview of the approach

\section{A DSL for Web Task Composition}

Procedures will be defined according with the DSL metamodel shown in Figure by a UML class model. This metamodel defines those elements contemplated by the DSL and their relations. Basically, the DSL defines a procedure as a XML file containing a list of tasks. Primitive tasks supported are based on [3]. The set of augmenters depends on what was developed by users. Composed tasks are used to group several tasks in a single block. Tasks have three main properties: repetition property for specifying if the task may be executed more than once. The optional property allows skipping the execution of the task. If automatic property is true, then the player automatically triggers the task. 


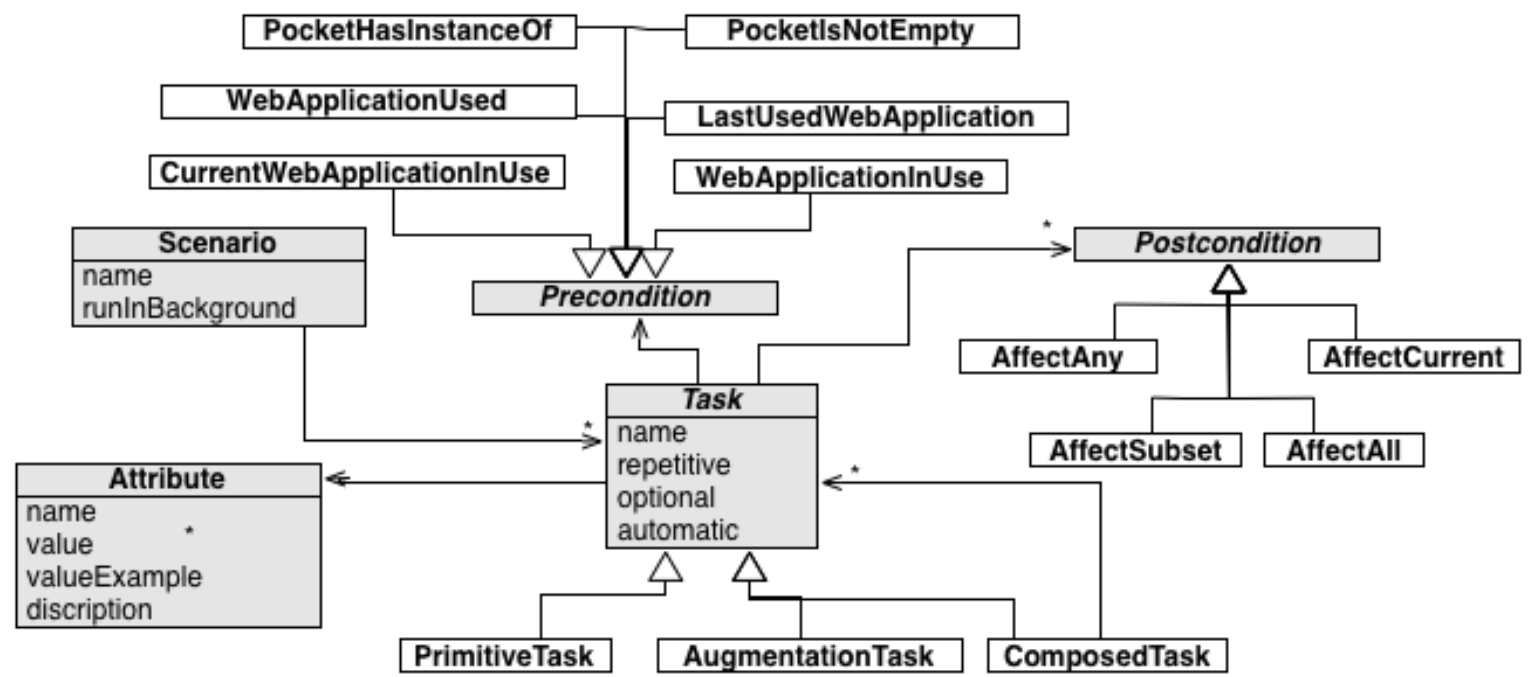

Fig. 4. The DSL metamodel

Besides these properties, for each task additional properties can be added including preconditions, postconditions and attributes:

- Preconditions: preconditions are used to decide if the task will be executed or not according to which information is available. There are two main kinds of preconditions. On the one side, preconditions about collected data: for conditioning the execution of a task according to the collected data. On the other side, preconditions about navigational history: for conditioning the execution of a task according to the Web applications used.

- Post-conditions: post-conditions are specified to determine the effect of executing a particular task. For example, AffectCurrent is used to specify that the execution will modify the current Web site.

- Attributes: refer to data required to accomplish tasks. Attributes (with name, values, etc.) are specified as metadata for each task.

ComposedTask allows creating dependencies in the DSL. With this kind of task a finite sequence of tasks can be manage altogether in order to mark as repetitive or optional this entire block.

In the example from Figure 3, we have used both pre and post conditions. For example in the augmentation task IconifiedLink we have specified the AffectSubset precondition with a regular expression that matches with all Wikipedia articles. In this way, when a new "PoI" is collected, all Wikipedia articles will be adapted by adding the corresponding link to Google Maps (the focused Wikipedia article and any other opened in non-focused Browser tabs). In order to show an example of precondition, we have used the PocketHasInstanceOf one in order to execute the augmenter only if an instance of "PoI" was collected.

\section{Tool Support}

We have developed two tools: an editor for creating procedures and a procedure player for parsing and executing procedures. 


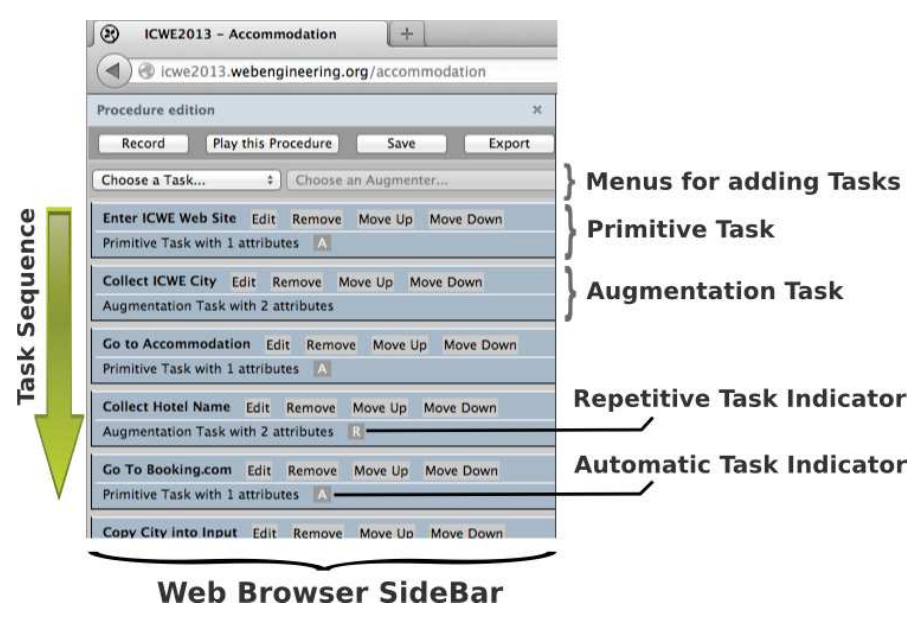

Fig 5.a. General view of the tool

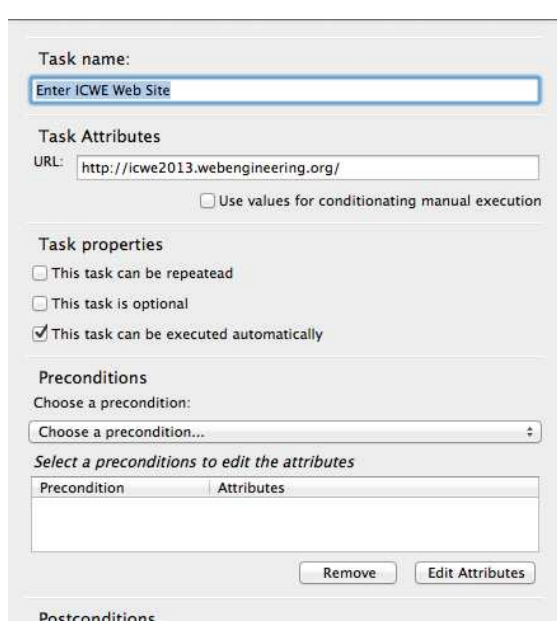

Fig. 5.b. Edition of a single task

Figure 5.a shows the editor: a sidebar that allows users to specify tasks into the procedure while analyzing Web sites. The tool provides an assisted mode: users may record their interaction with the Web and the corresponding tasks will be added to the procedure automatically. This mode contemplates both primitive and augmentation tasks. Figure 5.b shows how to edit a task. It allows users to specify the name, preand post-conditions as well as values for both properties and attributes.

The Procedure Player is shown in Figure 6. When the user selects a procedure to be executed, this appears in the Procedure Player. Once it is running, the Procedure Player may execute automatically a task (if the tasks was marked as automatic). Those tasks that have been executed appear with different styles, in order to give visual feedback to users when a task was finished. For manual tasks the Procedure Player waits to the corresponding user interaction. When this happens the task state changes and the following task in the sequence is executed. When the procedure has finished, the user may share the procedure execution (which includes both tasks definition and data used in each task) for future executions or even for share with partners.

\subsection{A Simple Case Study Using the Tools}

Figure 6 shows the execution of a procedure for planning a trip to ICWE2013. The first task "Enter ICWE Web Site" is automatic and it loads the ICWE2013 Web site. Then the procedure waits a manual task, which require from users to collect a City into the Pocket. Once it is made, the procedure loads the accommodation page.

The task "Collect Hotel name" allows user to collect hotel names. After that, an automatic task opens the site booking.com for searching rooms. Figure 6.b shows the booking.com loaded with the "Destination" input filled with the city previously collected. The procedure follows with augmenters for highlighting the selected hotels. 


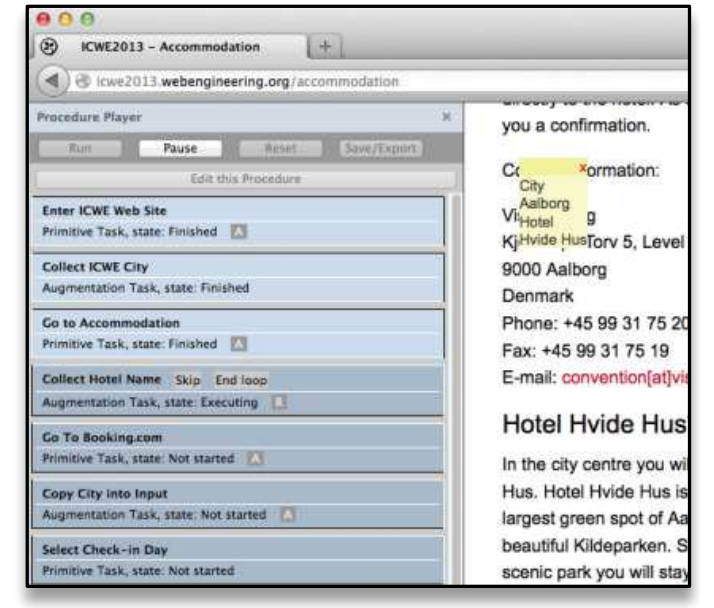

Fig. 6.a. Task Execution: collecting accommodation

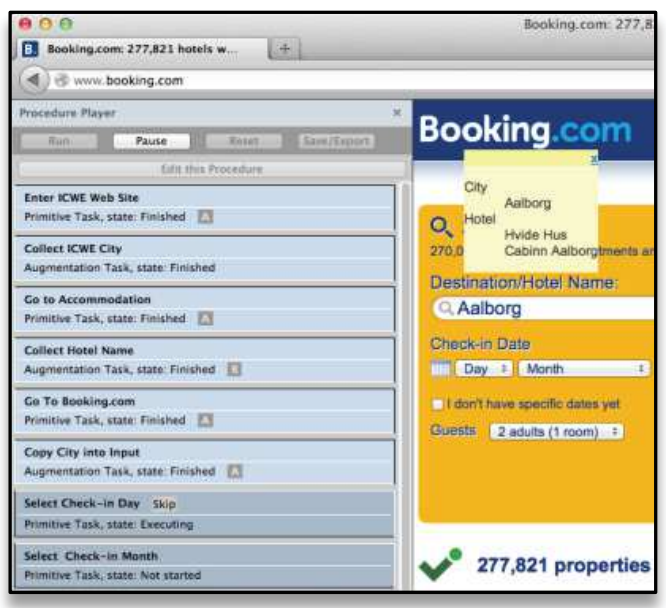

Fig. 6.b. Task Execution: looking for hotels rooms

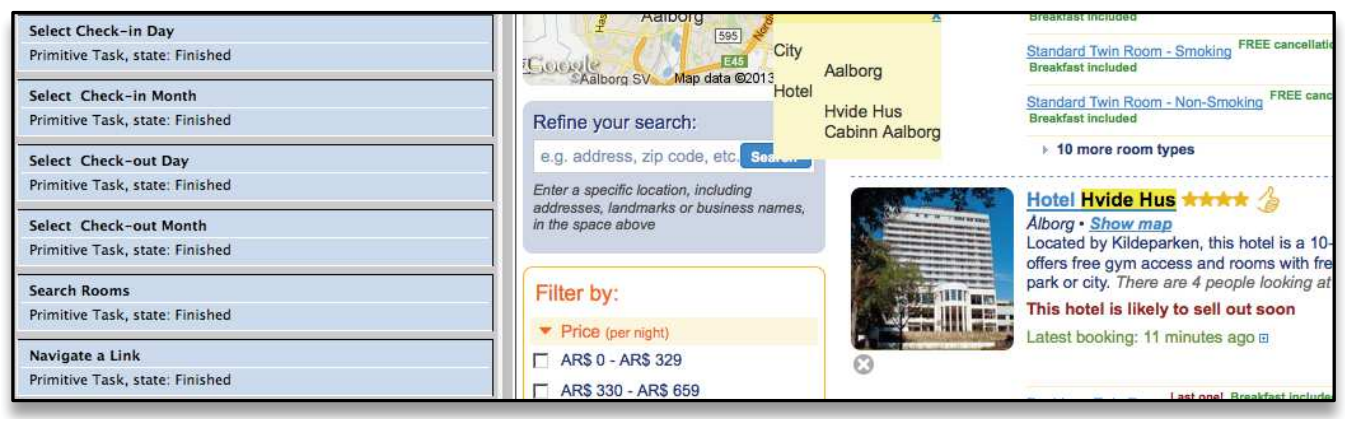

Fig. 7. Trip to ICWE procedure execution: searching and highlighting collected hotels

Figure 7 shows the procedure state once the user has finished several primitive tasks for searching for rooms. Once the results are shown, the hotel names collected are used by the task "Highlight collected Hotels" which adapt the current Web page for highlighting the relevant hotels. Once the hotel room payment is finished, the procedure gives the same support for buying flight tickets: it opens expedia.com, prefills the forms for search (it uses the geolocation component provided by the framework), etc. since some tasks are marked as automatic. Finally, it supports to the user in the task of filling forms with his personal data.

\subsection{Evaluation}

We have evaluated empirically the approach by performing the same task in different ways: manually, automatically with other tool (Selenium) and with procedures. We defined three procedures with different levels of automation: i) repeating the task structure but reentering all information, ii) repeating task structure and reusing information from previous execution, iii) fully automated. We assessed quantitatively the interactions made by the user using GOMS-Keystroke (KLM) model [6]. The GOMS-Keystroke (KLM) allows to simulate the performance of a trained user 
proposing the average time to perform basic action (for instance, reach for mouse takes $0,40 \mathrm{sec}$ ). Thus, provided a detailed scenario of user actions including low-level user actions, it is possible to estimate user performance (i.e. speed).

The task was Planning a Trip to ICWE, which implied to use three different Web sites: i) ICWE2013 home page to get information about the conference; ii) Expedia.com to buy flights tickets; and iii) boking.com to book a room in one of the conference hotel.

Table 1 summarizes the results obtained with each approach. The task was decomposed into smaller ones in order to show when the use of a tool makes the difference. A first task, Create Artifact, is only valid when a tool for automating tasks is used.

Table 1. Results of the evaluation

\begin{tabular}{|c|c|c|c|c|c|}
\hline \multirow[b]{2}{*}{ Task } & \multirow[b]{2}{*}{$\begin{array}{l}\text { Normal } \\
\text { Use }\end{array}$} & \multirow[b]{2}{*}{ Selenium } & \multicolumn{3}{|c|}{ Procedures } \\
\hline & & & $\begin{array}{c}\text { Semi } \\
\text { automatic }\end{array}$ & $\begin{array}{l}\text { Semi automatic with } \\
\text { data reutilization }\end{array}$ & Automatic \\
\hline Create Artifact & - & 9,5 & - & 472,2 & - \\
\hline Execute Artifact & - & - & 9,5 & 9,5 & 9,5 \\
\hline Get information about the conference & 12 & 9,5 & 14,2 & 14,2 & 0 \\
\hline Search Flights & 35,9 & \multirow[b]{4}{*}{9,5} & 1,7 & 1,7 & 0 \\
\hline Select Flights & 5 & & 6.3 & 6.3 & 0 \\
\hline Enter Passenger Information & 25,5 & & 25,5 & 0 & 0 \\
\hline Pay Flights & 59,7 & & 59,7 & 1,7 & 0 \\
\hline Search Room & 19,9 & \multirow[b]{4}{*}{9,5} & 3,6 & 3,6 & 0 \\
\hline Select Room & 6,5 & & 5,1 & 5,1 & 0 \\
\hline Enter Passenger Information & 29,4 & & 27,5 & 0 & 0 \\
\hline Pay Room & 51,4 & & 51 & 4,8 & 0 \\
\hline Total & 245,3 & 28.5 & 202,8 & 46,9 & 9,5 \\
\hline
\end{tabular}

Table also shows how much time was necessary with each approach. The most time consuming was the normal use $(245,3 \mathrm{~s})$. Selenium consumed $28.5 \mathrm{~s}$. The automatic procedure was the fastest. However it can be counterproductive since users lose the control over task. Semi-automatic execution only reproduced automatically those aspects like prefilling forms, and opening URLs when the previous task is finished, etc. Semi-automatic execution with data reutilization implies more automation by reusing data used in previous executions of the procedure such as prefilling forms with passenger information, credit card information, etc. In this case each confirmation steps (i.e. clicking search buttons) were performed manually. Finally, the fullautomated procedure performs even these last actions, but leaving the user unable to control the task. Defining the procedure took $472,2 \mathrm{sec}$. This time would be lower/higher accordingly to the automation level used. We only measured the case we thought was the best choice in our approach.

\section{Conclusions and Future Work}

We presented an approach and DSL for orchestrating user tasks over the Web. The approach allows easy integration of client-side scripts to build procedures that can be share with other users. The DSL provides a certain level of abstract that could be used 
to analyze the sequences of users' tasks used in procedures compositions. Each task may be pre-conditioned, and the data is not fixed a priori (the approach contemplates data collection as tasks); which gives flexibility. Manual tasks are contemplated too, in order to give control to users who may feel uncomfortable if the whole task is delegated in an automatic tool.

The case study presented shows that the tools are completely functional. An empiric evaluation shows how the approach improves the performance in the execution of complex tasks. However we need additional studies to explore the usability and potential of user adoption of such tools. In addition with user testing of the tools, future work will address the possibility of having synchronous communication between users performing procedures. Our ultimate goal is to allow users who create and share procedures with friends, be able to follow the execution of the procedures.

The approach opens up the way for potential collaboration between users. By sharing procedures or even synchronize users' procedures execution would allow users to collaborate in order to accomplish a task altogether or even to share a procedure execution with a partner.

\section{References}

1. Bolin, M., Webber, M., et al.: Automation and customization of rendered web pages. In: UIST 2005, pp. 163-172. ACM Press (2005)

2. Card, S., Moran, T., Newell, A.: The psychology of human-computer interaction, p. 448. Lawrence Erlbaum Associates, Hillsdale (1983)

3. Byrne, M.D., John, B., Wehrle, N., Crow, D.: The tangled Web we wove: a taskonomy of WWW use. In: Proc. of Conf. on Human factors in computing systems (CHI 1999), pp. 544-551. ACM, New York (1999)

4. Firmenich, S., Winckler, M., Rossi, G., Gordillo, S.: A Framework for Concern-Sensitive, Client-Side Adaptation. In: Auer, S., Díaz, O., Papadopoulos, G.A. (eds.) ICWE 2011. LNCS, vol. 6757, pp. 198-213. Springer, Heidelberg (2011)

5. Leshed, G., Haber, E., Matthews, T., Lau, T.: CoScripter: automating \& sharing how-to knowledge in the enterprise. In: Proc. of ACM SIGCHI 2008, pp. 1719-1728. ACM Press (2008)

6. Morris, M.R.: A survey of collaborative web search practices. In: Proc. of ACM SIGCHI 2008, pp. 1657-1660. ACM Press (2008)

7. Selenium, http://jroller.com/selenium/ (last visit: February 26, 2013)

8. Yu, J., Benatallah, B., Casati, F., Daniel, F.: Understanding Mashup Development. IEEE Internet Computing 12, 44-52 (2008) 